\section{Second-line anti-tuberculosis drug concentrations for susceptibility testing in} the MODS assay

\author{
Sean Patrick Fitzwater*,\#, G. Andrew Sechler*, , Oswaldo Jave ${ }^{+}$, Jorge Coronel*, \\ Alberto Mendoza ${ }^{\S}$, Robert H. Gilman`, Jon S. Friedland ${ }^{\dagger}$ and David A.J. Moore ${ }^{\star, \#, f, * *}$
}

ABSTRACT: Multidrug-resistant tuberculosis (TB) threatens TB control worldwide. The microscopic observation drug susceptibility (MODS) assay is a low-cost, high-performance TB diagnostic tool for rapid liquid culture and direct isoniazid and rifampicin drug susceptibility testing (DST). The objective of this study was to explore the potential for extending the MODS assay to rapid second-line DST and to identify critical concentrations of candidate drugs for prospective testing.

Sputum samples from 94 TB culture-positive patients receiving second-line TB agents were cultured following standardised MODS protocols, with a range of titrations of antimicrobial drugs added. Critical concentrations were determined using a modified Kaplan-Meier survival curve analysis.

Candidate critical concentrations were determined for capreomycin $\left(10 \mu \mathrm{g} \cdot \mathrm{mL}^{-1}\right)$, ciprofloxacin $\left(1.25 \mu \mathrm{g} \cdot \mathrm{mL}^{-1}\right)$, cycloserine $\left(40 \mu \mathrm{g} \cdot \mathrm{mL}^{-1}\right)$, ethambutol $\left(10 \mu \mathrm{g} \cdot \mathrm{mL}^{-1}\right)$, ethionamide $\left(5 \mu \mathrm{g} \cdot \mathrm{mL}^{-1}\right)$, kanamycin $\left(5 \mu \mathrm{g} \cdot \mathrm{mL}^{-1}\right)$, para-aminosalicylic acid $\left(10 \mu \mathrm{g} \cdot \mathrm{mL}^{-1}\right)$ and streptomycin $\left(10 \mu \mathrm{g} \cdot \mathrm{mL}^{-1}\right)$. No cut-off point was identified for the other second-line drugs or for pyrazinamide.

At particular concentrations of some second-line TB drugs this novel Kaplan-Meier analysis clearly differentiated populations that were susceptible or resistant. These candidate critical concentrations should now be tested in a range of epidemiological settings to define the performance of direct, second-line TB DST with MODS, offering potential low-cost second-line TB DST capacity.

KEYWORDS: Diagnostics, extensively drug-resistant tuberculosis, multidrug resistance, secondline drug susceptibility testing, tuberculosis

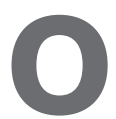
ver 1.7 million deaths are attributed to tuberculosis (TB) annually, the leading curable cause of death from infectious disease in the world [1]. The impact of TB is greatest in resource-constrained settings, where the poor suffer disproportionately. Despite progress towards the targets set out by the United Nations Millennium Development Goals and the Stop TB Partnership, the expansion of robust TB diagnostic capacity remains a challenge $[1,2]$.

Drug-resistant TB threatens progress towards TB control $[1,2]$. Globally the burden of multidrugresistant (MDR) TB is estimated at 440000 cases, and extensively drug-resistant (XDR) TB has been found in 58 countries $[1,2]$. Estimates of XDR-TB prevalence are, in part, limited by the availability of drug susceptibility testing (DST) for secondline TB drugs. However, XDR-TB accounts for $>10 \%$ of MDR-TB cases in the former Soviet
Union and South Africa, where the potentially devastating impact of XDR-TB-HIV co-infection has been demonstrated [2-6].

The emergence of XDR-TB is an indirect indication of programmatic failure to adequately diagnose and treat MDR-TB [7]. Wider availability of rapid second-line DST that performs reliably and cost-effectively in resource-limited settings is needed to address this threat $[8,9]$. The nonproprietary microscopic observation drug susceptibility (MODS) assay, a high-performance diagnostic technique for the liquid culture-based detection of TB and direct DST for MDR-TB, has the potential to address this need. Developed in Peru, MODS has been shown to be a rapid, reliable, low-cost method for DST of isoniazid and rifampicin, but studies have not yet assessed performance in direct DST for second-line drugs [10]. Additionally, data on ethambutol (EMB),
AFFILIATIONS

*Laboratorio de Investigación de Enfermedades Infecciosas, Universidad Peruana Cayetano Heredia, Lima,

+Estrategia Sanitaria Nacional de Prevención y Control de la

Tuberculosis, Ministerio de Salud, Lima, and

${ }^{\S}$ Laboratorio de Referencia Nacional de Mycobacteria, Instituto Nacional de Salud (INS), Lima, Peru.

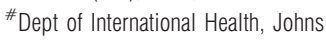
Hopkins Bloomberg School of Public Health, Baltimore, MD, and "Dept of Internal Medicine and Paediatrics, Mt Sinai School of Medicine, New York, NY, USA. ${ }^{f}$ Dept of Infectious Diseases and Immunity and Wellcome Centre for Clinical Tropical Medicine, Imperial College London, London, and **LSHTM TB Centre and Dept of Clinical Research, Faculty of Infectious and Tropical Diseases, London School of Hygiene and Tropical Medicine, London, UK.

CORRESPONDENCE

D.A.J. Moore

Dept of Clinical Research, Faculty of Infectious and Tropical Diseases London School of Hygiene and Tropical Medicine Keppel Street London WC1E 7HT UK E-mail: David.Moore@Ishtm.ac.uk

Received:

April 102012

Accepted after revision: Aug 022012 First published online: Aug 162012 
streptomycin (STM) and pyrazinamide (PZA) DST in MODS have failed to clearly demonstrate high performance at the drug concentrations employed [10-12].

Evaluation of MODS performance for direct DST for these three drugs and for second-line agents will require a large, prospective, multicentre study comparing MODS with existing reference standards in a range of epidemiological settings. Because MODS is a direct drug susceptibility test, the evaluation needs to be performed using fresh sputum samples, not a bank of stored strains with known DST profiles. In order to design such a trial it is necessary to first define the candidate critical concentrations for each drug, which was the primary goal of this study. Using the receiver operating curve (ROC) methodology [13] and a novel Kaplan-Meier survival curve analysis, we aimed to explore the inhibitory capability of a wide range of concentrations of each of the drugs tested and to define the concentrations in MODS at which strains defined as susceptible or resistant by the reference standard were best differentiated. Thus, identified candidate critical concentrations may then be prospectively evaluated in a larger trial as described above.

\section{MATERIALS AND METHODS \\ Field methods}

Candidate participants with confirmed MDR-TB were identified from databases of the Peruvian Instituto Nacional de Salud (INS; Lima, Peru) and selected for inclusion if they were scheduled to receive specific MDR therapy, the most recent sputum culture was positive and resistance to any second-line agent had been demonstrated previously. Consenting participants provided a sputum sample of $\geqslant 5 \mathrm{~mL}$, which was transferred to the Universidad Peruana Cayetano Heredia (UPCH) laboratory in Lima, where it was stored refrigerated and processed, generally within $24 \mathrm{~h}$ of collection.

\section{Laboratory methods}

Sputum samples were processed according to the MODS methodology [14], with modifications to incorporate seven different concentrations of capreomycin (CAP), ciprofloxacin $(\mathrm{CIP})$, cycloserine $(\mathrm{CS}), \mathrm{EMB}$, ethionamide (ETO), kanamycin (KM), para-aminosalicylic acid (PAS), PZA and STM. Sputum samples were decontaminated by the $\mathrm{NaOH}-\mathrm{N}$-acetyl-L-cysteine method and reconstituted in supplemented Middlebrook 7H9 broth (Becton Dickinson, Franklin Lakes, NJ, USA) as described previously $[15,16] .540 \mu \mathrm{L}$ of sample-broth mixture was pipetted into each well of three 24-well plates. $60 \mu \mathrm{L}$ of preformulated antibiotic was added to a series of seven wells to achieve the desired range of in-well antibiotic concentrations, and an eighth well was a drug-free control.

The range of antibiotic concentrations that were tested in this study was based on previous studies [13, 17-24]. The following ranges were tested: CAP and ETO: $0.3125-20 \mu \mathrm{g} \cdot \mathrm{mL}^{-1}$; CIP: 0.1567-10 $\mu \mathrm{g} \cdot \mathrm{mL}^{-1}$; EMB, KM and STM: $0.625-40 \mu \mathrm{g} \cdot \mathrm{mL}^{-1}$; and PZA: $12.5-800 \mu \mathrm{g} \cdot \mathrm{mL}^{-1}$. Early experiments indicated a need to increase the upper limit of CS and PAS to the following ranges: CS: $0.3125-20 \mu \mathrm{g} \cdot \mathrm{mL}^{-1}$ initially, later $5-320 \mu \mathrm{g} \cdot \mathrm{mL}^{-1}$; PAS: $0.1567-10 \mu \mathrm{g} \cdot \mathrm{mL}^{-1}$ initially, later $0.625-40 \mu \mathrm{g} \cdot \mathrm{mL}^{-1}$. PZA experiments were initially carried out at $\mathrm{pH} 6.8$ (as for all other drugs) and $\mathrm{pH} 6.0$ because PZA activity is dependent upon an acidic $\mathrm{pH}$. However, Mycobacterium tuberculosis growth in drug-free wells was significantly impaired by the lower $\mathrm{pH}$. Subsequent PZA experiments were limited to $\mathrm{pH} 6.8$.

Inoculated plates were incubated and examined for growth as previously described $[11,25,26]$. Once TB growth was observed in a drug-free well, all other wells were examined. In the event of contamination or inconsistent growth, the original sample was decontaminated again and re-cultured. M. tuberculosis was confirmed using spoligotyping or PCR [27]. Inconsistent growth was defined as $M$. tuberculosis growth in a well with a higher drug concentration than a well that showed no growth. Isolates were sent for reference first- and secondline DST by the proportion method at the Peruvian National Mycobacteria Reference Laboratory (at INS, Lima).

\section{Statistical analysis}

Data were analysed using Stata 9.1 (StataCorp, College Station, TX, USA) and Excel 2003 (Microsoft; Microsoft Corp., Redmond, WA, USA). The sensitivity and specificity of the differing concentrations was calculated using the proportion method as the reference. ROC curves were constructed from this data. A Kaplan-Meier survival analysis was performed using increasing drug concentrations in place of the usual time variable. The survival failure event for each series was the point at which a sample yielded a positive culture at all lower drug concentrations, but not higher concentrations. Series with growth at the highest drug concentration were censored at that point. Individual drug concentration series that did not follow this pattern due to inconsistent growth or contamination at critical concentrations were withheld from the analysis. Critical concentrations for each drug were chosen from the KaplanMeier survival curves by identifying the junction point between the rapid sterilisation phase of the curves and the more level later portions of the curves. Curves that did not follow the expected pattern were stratified by auramine smear status and proportion method resistance. The results of the ROC and survival analyses were compared to determine the most appropriate candidate critical concentration.

\section{Ethical review}

The institutional review board of UPCH (Lima) approved the study protocol and the informed consent form, and the National TB Control Programme at the Peruvian Ministry of Health, Lima, granted technical approval.

\section{RESULTS}

\section{Study population baseline characteristics}

115 patients identified from the databases of the MDR Technical Unit (UT-MDR) of the Peruvian National TB Control Programme and the Peruvian INS agreed to participate and were able to give sufficient samples to be tested. 94 of these were culture-positive in MODS, $65 \%$ of which were also auramine smear-positive. The number of drugs to which resistance, as determined by proportion method, was identified in a single sample ranged from three to nine, with an average of resistance to six drugs per sample (table 1); 88 (94\%) samples were MDR.

The 94 samples that grew M. tuberculosis had 822 dilution series performed on them, which included repeated dilutions due to contamination and altered drug concentrations. Of the dilution series, $25(3 \%)$ were not included in the survival 
analysis because they were considered inconclusive due to poor growth or contamination. Complete drug titration series data were obtained for all nine drugs in 85 (90.4\%) samples. In total, 7293 MODS wells were cultured, with 54 (0.7\%) unreadable due to contamination.

\section{ROC curves}

Using proportion method results as the reference standard, ROC curves were derived for each drug (fig. 1). For CIP, KM and PAS, but none of the other drugs, it was possible to identify single drug concentrations that delivered both good sensitivity and specificity in the detection of (proportionmethod defined) resistance. As a result, the ROC curves played a limited role in determining critical concentrations.

\section{Kaplan-Meier survival curves}

The Kaplan-Meier survival curves for CIP, CS, EMB, KM and PAS (fig. 2) followed the expected S-shaped curve, showing little growth inhibition at low drug concentrations, followed by a steep decline in survival probability where presumably drug susceptible $M$. tuberculosis are killed, and ending with a levelling off where resistant organisms are able to survive at higher drug concentrations. Candidate critical drug concentrations (table 2) were taken directly from these curves. Stratification by auramine sputum smear status did not demonstrate any discernable difference in curve shape for any of these agents (data not shown).

In contrast, the Kaplan-Meier survival curves for ETO and STM demonstrated differential dynamics in response to increased drug concentrations when smear-positive and smear-negative samples were considered separately (fig. 3), with smear-positive samples requiring higher drug concentrations to inhibit growth (but no incremental effect with increasing grade of smear-positivity; data not shown). All other drugs were stratified in this manner, but no effect of smear status was seen. Candidate critical concentrations selected for these drugs (table 2) were determined from the smear-negative curve to minimise misclassification of drugresistant smear-negative samples.

For CAP a linear relationship between drug concentration and probability of culture-positivity was demonstrated (fig. 2). A sub-analysis separately evaluating samples deemed susceptible or resistant by the proportion method and then further stratified by smear status is shown in figure 4 . All other drugs were stratified in this manner, but no effect of smear or drug resistance status was seen. Among samples designated as susceptible by the proportion method there appears to be a sub-population of around $25 \%$ of strains from this patient cohort in Lima which resist high concentrations of CAP $\left(>10 \mu \mathrm{g} \cdot \mathrm{mL}^{-1}\right)$ in MODS.

The Kaplan-Meier survival curves for PZA showed limited, linear response to increased drug concentrations. Stratification by smear status and/or proportion method result did not improve the capacity to distinguish distinct populations with different susceptibility.

Candidate critical concentrations derived from these curves and the concordance of MODS using these concentrations with the proportion method in this study are shown in table 2. Except for EMB, STM and ETO, for which performance is poor, and PZA, for which no candidate critical concentration could be derived, the concordance of direct MODS second-line DST using the candidate concentrations identified with proportion method DST was $>75 \%$ for all agents tested, and as high as $89 \%$ for KM.

\section{DISCUSSION}

These data demonstrate that candidate critical concentrations, which now demand prospective evaluation in multiple different epidemiological circumstances, can be derived for most secondline anti-TB drugs in the MODS assay. The results expand the range of MODS to several additional agents using the following concentrations: CAP: $10 \mu \mathrm{g} \cdot \mathrm{mL}^{-1}$; CIP: $1.25 \mu \mathrm{g} \cdot \mathrm{mL}^{-1}$; CS: $40 \mu \mathrm{g} \cdot \mathrm{mL}^{-1}$; EMB: $10 \mu \mathrm{g} \cdot \mathrm{mL}^{-1}$; ETO: $5 \mu \mathrm{g} \cdot \mathrm{mL}^{-1}$; $\mathrm{KM}: 5 \mu \mathrm{g} \cdot \mathrm{mL}^{-1}$; PAS: $10 \mu \mathrm{g} \cdot \mathrm{mL}^{-1}$ and STM: $10 \mu \mathrm{g} \cdot \mathrm{mL}^{-1}$. The critical concentration for CS should be considered preliminary, due to the limited number of resistant strains identified, and no appropriate concentration could be found for PZA.

An important strength of this study was the availability of clinical sputum samples from patients with variable exposure histories to second-line agents and well-characterised DST profiles, including a high proportion of MDR specimens. The use of clinical samples rather than strains is important for direct DST methods and the patient group studied (MDR with second-line drug exposure) is precisely the first clinical group that might be targeted by MODS second-line DST were it available. However, because the samples show a rather high level of drug resistance, it is not certain whether the critical concentrations described here are relevant to samples with lower levels of resistance. Further testing with strains that demonstrate a lower degree of resistance would add to the generalisability of the results demonstrated in this study. Additionally, 19 patients who were culture positive in the UTMDR database were found to be culture negative on sampling, probably due to treatment prior to sampling for this study, and

\begin{tabular}{|c|c|}
\hline Drug & Resistance $^{\#}$ \\
\hline \multicolumn{2}{|l|}{ First-line } \\
\hline Ethambutol & $55(59)$ \\
\hline Isoniazid & $94(100)$ \\
\hline Pyrazinamide & $40(44)$ \\
\hline Rifampicin & $88(94)$ \\
\hline Streptomycin & $62(66)$ \\
\hline Multidrug resistant (total) & $88(94)$ \\
\hline \multicolumn{2}{|l|}{ Second-line } \\
\hline Capreomycin & $51(54)$ \\
\hline Ciprofloxacin & $34(36)$ \\
\hline Cycloserine & $5(5)$ \\
\hline Ethionamide & $51(54)$ \\
\hline Kanamycin & $70(75)$ \\
\hline Para-aminosalicylic acid & $18(19)$ \\
\hline
\end{tabular}



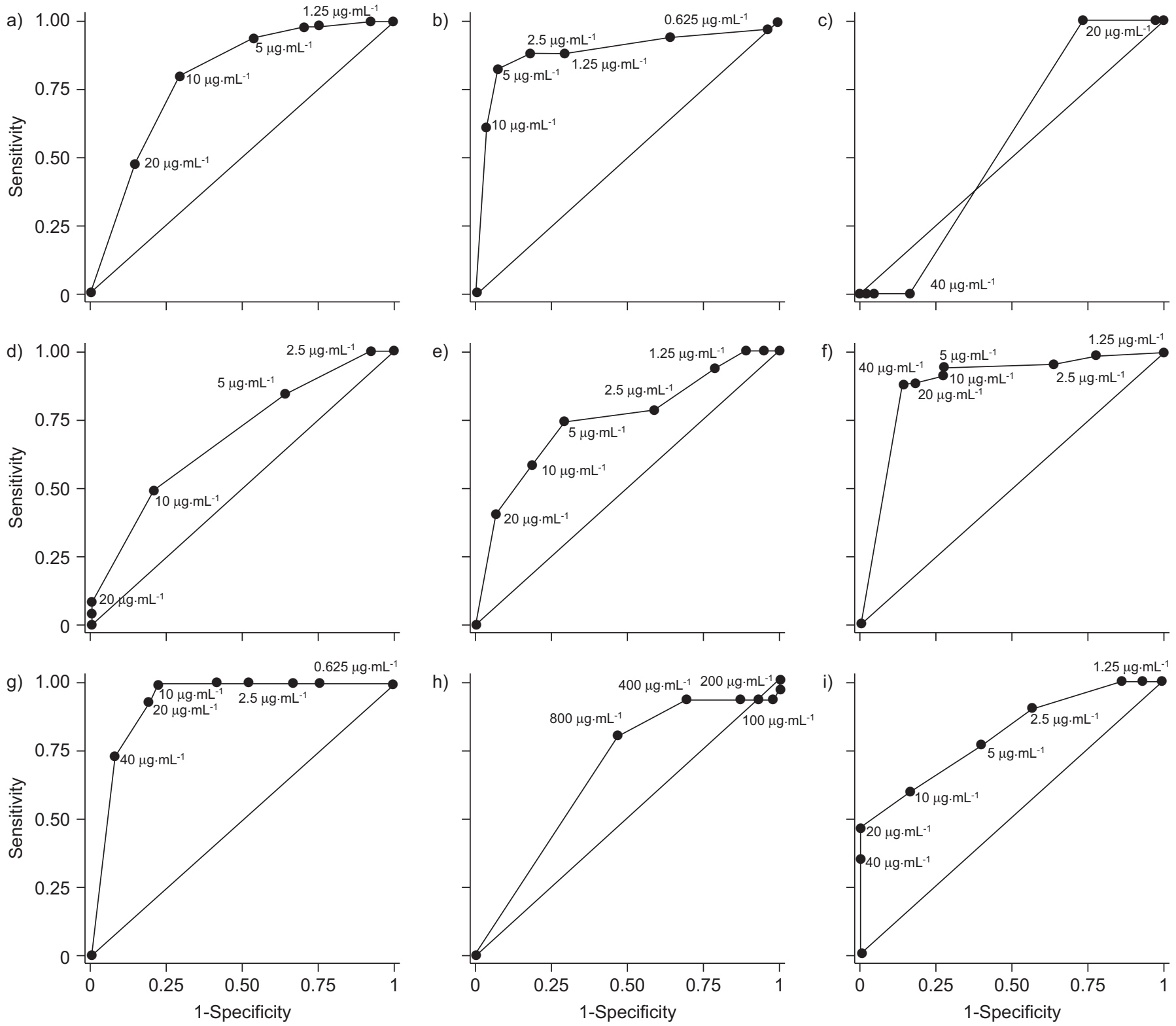

FIGURE 1. Receiver operating characteristic curves for a) capreomycin, b) ciprofloxacin, c) cycloserine, d) ethambutol, e) ethionamide, f) kanamycin, g) paraaminosalicylic acid, h) pyrazinamide and i) streptomycin. Curves that reach furthest towards the top left-hand corner indicate potential MODS (microscopic observation drug susceptibility) well drug concentrations with both high sensitivity and specificity for the detection of resistance to that drug.

six patients who were reported to have MDR-TB in the UTMDR database were found to not have rifampicin resistance when the proportion method was later used to confirm the resistance pattern. It is not certain whether this discrepancy is due to shifts in strain resistance, or laboratory variability.

These study data are timely, as in this era of roll-out of molecular testing for rifampicin resistance there is likely to be a growing demand for phenotypic second-line DST; this is currently limited to Mycobacteria Growth Indicator Tube (MGIT; Becton Dickinson) culture and DST, which is expensive and largely only available at national reference laboratory level. Notwithstanding remediable teething troubles with rifampicin DST by Xpert MTB/RIF, the GeneXpert platform (Cepheid, Sunnydale, CA, USA) offers considerable promise for future, wider molecular DST. However, resistance to many of these agents (including isoniazid) is complex, and there is a large and important knowledge gap in recognising all mutations responsible for phenotypic resistance for each drug, suggesting that molecular testing alone is unlikely to be sufficient in the foreseeable future. If MODS was proven to be reliable for quinolone and injectable second-line agent DST, it would provide another alternative option for TB laboratory networks seeking to manage increasing numbers of patients identified with rifampicin and/or isoniazid resistance.

Proportion method-defined resistance to individual secondline agents has never been proven to be associated with clinical failure, and such outcome data will probably remain an elusive goal. Proportion method cut-off points are defined by 

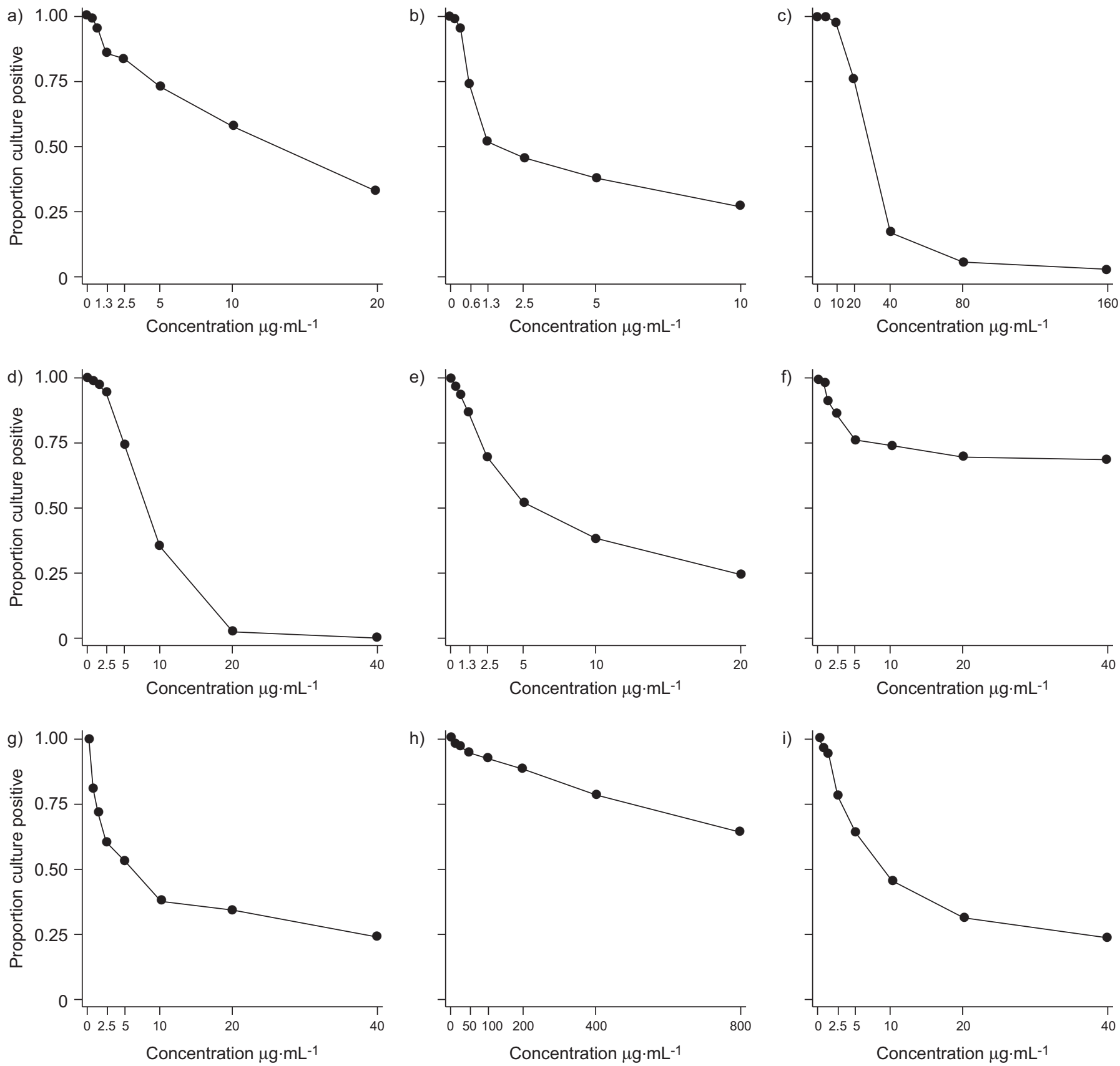

FIGURE 2. Kaplan-Meier survival curves for the proportion of positive culture in the presence of increasing concentrations of drug: a) capreomycin, b) ciprofloxacin, c) cycloserine, d) ethambutol, e) ethionamide, f) kanamycin, g) para-aminosalicylic acid, h) pyrazinamide and i) streptomycin.

comparing the growth of strains derived from patients with either no or extensive previous treatment with the agent of interest. The proportion method also allows for the breakthrough growth of some bacilli in the presence of the drug tested; provided they represent $<1 \%$ of all those present, the sample will be designated as drug susceptible, under the assumption that these remaining bacteria will be sterilised by the other drugs $[28,29]$. MODS does not make this assumption; any growth is considered positive, and therefore resistant to the drug.

Because of these shortcomings in the putative reference standards for determining second-line $M$. tuberculosis resistance status and the high degree of variability often seen between drug susceptibility methods [24, 30-34], we used a modified survival analysis to examine the response of the strains within samples to increasing concentrations of drugs in the MODS assay. This allowed us to differentiate visually between populations of bacteria, based on their response to drug concentrations, giving context to the critical concentrations beyond a potentially flawed comparison to other imperfect drug susceptibility tests. However, it is important to note that even when populations are distinguished, the selection of a critical concentration depends upon the perspective of the viewer. In this study we generally selected lower drug concentrations to 


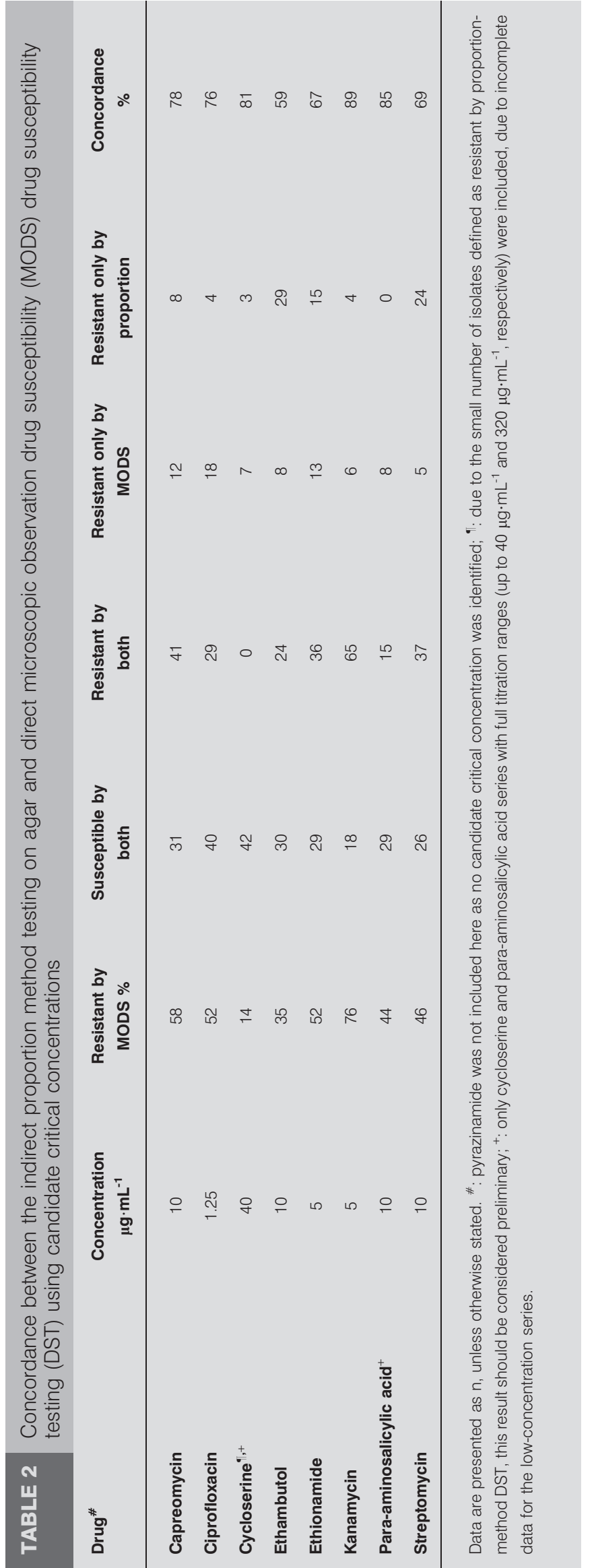

ensure that strains that showed an intermediate response to drug titrations were considered resistant, and thereby to minimise the risk of ineffective treatment.

Using the survival analysis we were also able to examine the effect of auramine smear status on the critical concentrations. This is important because, being a direct test, there is no control of inoculum size in MODS. For rifampicin and isoniazid this has now repeatedly been shown to be of no importance, but the same was not known previously for other agents. Here, auramine status had little effect on the overall shape of the survival curves for CIP, CS, EMB, KM and PAS, and therefore no implications for choosing the critical drug concentrations, as has been previously shown for rifampicin and isoniazid DST [11]. This potentially offers an important comparative advantage over molecular tools such as GenoType ${ }^{\circledR}$ MTBDRplus (Hain Lifescience, Nehren, Germany), which to date are only validated for use on smear-positive samples. However, auramine smear status did have an effect for CAP, ETO and STM. For these drugs the critical concentrations selected were based primarily on the auramine-negative curves, to minimise the chance that resistant strains are misclassified due to low mycobacterial counts in the sputum sample.

Even though our method for determining critical concentrations did not involve directly comparing the resistance as determined by proportion method, a high degree of agreement was generally seen between the two methods (table 2) and this
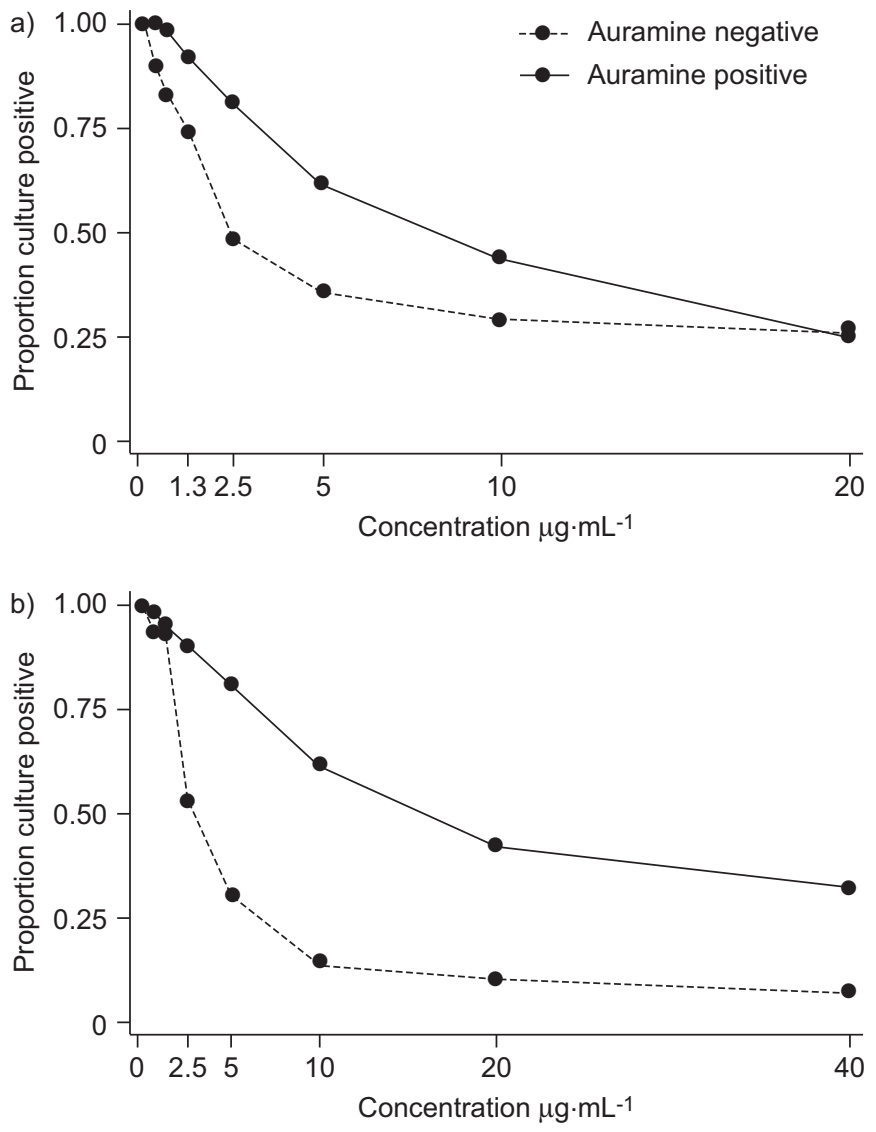

FIGURE 3. Kaplan-Meier survival curves stratified by auramine smear status for a) ethionamide and b) streptomycin. 

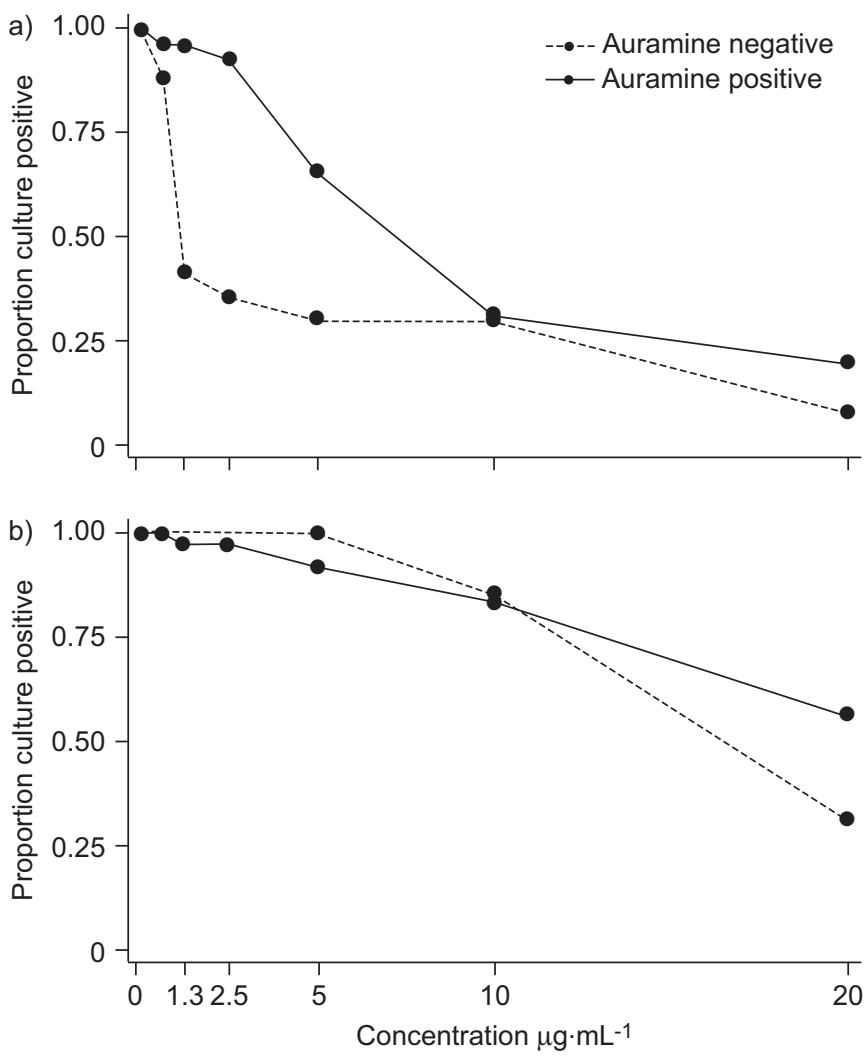

FIGURE 4. Kaplan-Meier survival curves for capreomycin of samples with strains classified as a) susceptible or b) resistant by the proportion method stratified by smear status.

was consistent with the results of the ROC curve comparisons (fig. 1).

Agreement between MODS and proportion method DST results for EMB and STM were poor. However, comparisons of proportion method with newer DST methods have often demonstrated poor agreement and wide variability between studies when examining these drugs, which has led to multiple revisions of the suggested minimum inhibitory concentration used in newer tests [35]. Studies of the MGIT960 platform have demonstrated sensitivities for detecting resistance as defined by the proportion method for EMB of $63-100 \%$, with sensitivities for detecting STM resistance of $77-100 \%$ [5, 24, 31]. Similar results have been observed for BACTEC 460 (Becton Dickinson) with sensitivities for resistance as defined by the proportion method of 25-91\% for EMB and 51-95\% for STM [5, 12, 24, 31, 32]. Comparison of MODS results for EMB and STM with a more reliable automated DST system may be necessary to validate the minimum inhibitory concentrations found in this study.

PZA DST is problematic because the drug is activated in acidic conditions, making an acidic culture $\mathrm{pH}$ a requirement for determining PZA-resistant phenotypes [36]. As with other culture methods [37, 38], the growth of $M$. tuberculosis in MODS culture is inhibited by acidic conditions ( $\mathrm{pH}$ 6.0) even without the addition of antimycobacterial agents. When performed at $\mathrm{pH} 6.8$ there was much better growth of M. tuberculosis, but the addition of PZA appeared to have little effect in distinguishing drug-susceptible from drug-resistant isolates. Clearly, further work is necessary to determine the appropriate conditions for using MODS as a drug susceptibility test for PZA.

CS has always been a difficult drug for which to find a critical concentration in other DST methods $[17,39]$. A recent study, which attempted to standardise DSTs, could not find acceptable critical concentrations for CS using the Middlebrook 7H10 agar or BACTEC 460, and proportion method testing was also unsatisfactory [39]. In this study, few isolates were found that were resistant to CS by either the proportion method or MODS criteria, and there were no isolates that were resistant by both methods. However, based on our survival curve analysis of CS, two populations were clearly distinguished, one which could be inhibited by CS, and one which grew despite high concentrations of CS. This suggests that a critical concentration of $40 \mu \mathrm{g} \cdot \mathrm{mL}^{-1}$ might be a reasonable preliminary estimate and a starting point for further evaluation.

In summary, this work has used a novel survival analysis approach to define candidate critical concentrations for secondline DST for M. tuberculosis using the rapid, low-cost, direct DST MODS platform. We were able to demonstrate differential responses to increasing drug concentrations using modified survival curves, which we believe to be an unbiased assessment of resistance. Further studies, perhaps with comparison to an automated system, would be beneficial to: 1) confirm the results found here; 2) determine if modified MODS culture conditions may permit identification of a PZA cut-off point; 3) further explore agents that showed relatively lesser concordance between the MODS and proportion methods; and 4) expand the DST repertoire to include a broader range of second-line drugs, such as amikacin, levofloxacin, moxifloxacin, linezolid, amoxicillin-clavulanate, delanamid and TMC207.

In this era of growing MDR/XDR-TB incidence the demand for affordable, reliable second-line DST to guide MDR treatment is becoming irresistible. Phenotypic testing currently outperforms genotypic testing because the full spectrum of resistanceconferring genetic mutations is, as yet, unknown for any agents. MODS offers the possibility of high-quality, reliable phenotypic testing in an affordable and adaptable format. Prospective evaluation of the performance of MODS for second-line DST using these critical concentrations in various study populations with a range of epidemiological characteristics and drug exposure histories will define how this tool for rapid, low-cost phenotypic DST should best be employed.

\section{SUPPORT STATEMENT}

This work received financial support from The Wellcome Trust (grant 084550/Z/07/Z) and The Director's Initiative Fund of TDR (UNICEF/ UNDP/World Bank/WHO Special Programme for Research and Training in Tropical Diseases). S. Fitzwater was supported by grant number T35AI065385 from the National Institute of Allergy And Infectious Diseases. D.A.J. Moore was supported by the Wellcome Trust.

\section{STATEMENT OF INTEREST}

None declared.

\section{ACKNOWLEDGEMENTS}

We wish to thank all study volunteers, without whose participation this work would have been impossible. The UPCH field and laboratory 
research team (Universidad Peruana Cayetano Heredia, Lima, Peru; P. Navarro, V. Huancaré, S. Lopez, R. Limascca, F. Garcia and C. Solis), Peruvian National Mycobacteria Laboratory team in Lima (L. Asencios, E. Leo and J. Ramírez) and Unidad Tecnica de TB-MDR (Ministerio de Salud, Lima) staff (R. Jamanca, A. Crossa and A.M. Chavez), who assisted in the significant logistical support required in order to ensure smooth running of the study.

\section{REFERENCES}

1 WHO Global Tuberculosis Control Report 2010. Geneva, World Health Organization, 2010.

2 Multidrug and Extensively Drug-resistant TB (M/XDR-TB): 2010 Global Report on Surveillance and Response. Geneva, World Health Organization, 2010.

3 Gandhi NR, Moll A, Sturm AW, et al. Extensively drug-resistant tuberculosis as a cause of death in patients co-infected with tuberculosis and HIV in a rural area of South Africa. Lancet 2006; 368: 1575-1580.

4 Andrews JR, Gandhi NR, Moodley P, et al. Exogenous reinfection as a cause of multidrug-resistant and extensively drug-resistant tuberculosis in rural South Africa. J Infect Dis 2008; 198: 1582-1589.

5 Andrews JR, Shah NS, Gandhi N, et al. Multidrug-resistant and extensively drug-resistant tuberculosis: implications for the HIV epidemic and antiretroviral therapy rollout in South Africa.J Infect Dis 2007; 196: Suppl. 3, S482-S490.

6 Koenig R. Drug-resistant tuberculosis. In South Africa, XDR TB and HIV prove a deadly combination. Science 2008; 319: 894-897.

7 Shah NS, Wright A, Bai GH, et al. Worldwide emergence of extensively drug-resistant tuberculosis. Emerg Infect Dis 2007; 13: 380-387.

8 Strategic and Technical Advisory Group for Tuberculosis: Report on Conclusions and Recommendations. Seventh Meeting. Geneva, World Health Organization, 2007.

9 Raviglione MC, Smith IM. XDR tuberculosis - implications for global public health. N Engl J Med 2007; 356: 656-659.

10 Moore DA, Evans CA, Gilman RH, et al. Microscopic-observation drug-susceptibility assay for the diagnosis of TB. N Engl J Med 2006; 355: 1539-1550.

11 Moore DA, Mendoza D, Gilman RH, et al. Microscopic observation drug susceptibility assay, a rapid, reliable diagnostic test for multidrug-resistant tuberculosis suitable for use in resource-poor settings. J Clin Microbiol 2004; 42: 4432-4437.

12 Madison B, Robinson-Dunn B, George I, et al. Multicenter evaluation of ethambutol susceptibility testing of Mycobacterium tuberculosis by agar proportion and radiometric methods. J Clin Microbiol 2002; 40: 3976-3979.

13 Luna-Herrera J, Martinez-Cabrera G, Parra-Maldonado R, et al. Use of receiver operating characteristic curves to assess the performance of a microdilution assay for determination of drug susceptibility of clinical isolates of Mycobacterium tuberculosis. Eur J Clin Microbiol Infect Dis 2003; 22: 21-27.

14 Brady MF, Coronel J, Gilman RH, et al. The MODS method for diagnosis of tuberculosis and multidrug resistant tuberculosis. $J$ Vis Exp 2008; 18: 845.

15 Laboratory Services in TB control. Parts I, II, and III. WHO/tb/ 98.258. Geneva, World Health Organization, 1998.

16 Kubica GP, Dye WE, Cohn ML, et al. Sputum digestion and decontamination with $\mathrm{N}$-acetyl-L-cysteine-sodium hydroxide for culture of mycobacteria. Am Rev Respir Dis 1963; 87: 775-779.

17 Pfyffer GE, Bonato DA, Ebrahimzadeh A, et al. Multicenter laboratory validation of susceptibility testing of Mycobacterium tuberculosis against classical second-line and newer antimicrobial drugs by using the radiometric BACTEC 460 technique and the proportion method with solid media. J Clin Microbiol 1999; 37: 3179-3186.
18 Bastian I, Rigouts L, Palomino JC, et al. Kanamycin susceptibility testing of Mycobacterium tuberculosis using Mycobacterium Growth Indicator Tube and a colorimetric method. Antimicrob Agents Chemother 2001; 45: 1934-1936.

19 Martin A, Camacho M, Portaels F, et al. Resazurin microtiter assay plate testing of Mycobacterium tuberculosis susceptibilities to second-line drugs: rapid, simple, and inexpensive method. Antimicrob Agents Chemother 2003; 47: 3616-3619.

20 Pracharktam R, Angkananukool K, Vibhagool A. In vitro susceptibility testing of levofloxacin and ofloxacin by microtiter plate Alamar blue against multidrug and non multidrug resistant Mycobacterium tuberculosis in Thailand. J Med Assoc Thai 2001; 84: 1241-1245.

21 Rusch-Gerdes S, Pfyffer GE, Casal M, et al. Multicenter laboratory validation of the BACTEC MGIT 960 technique for testing susceptibilities of Mycobacterium tuberculosis to classical secondline drugs and newer antimicrobials. J Clin Microbiol 2006; 44: 688-692.

22 Kruuner A, Yates MD, Drobniewski FA. Evaluation of MGIT 960based antimicrobial testing and determination of critical concentrations of first- and second-line antimicrobial drugs with drugresistant clinical strains of Mycobacterium tuberculosis. J Clin Microbiol 2006; 44: 811-818.

23 Caviedes L, Delgado J, Gilman RH. Tetrazolium microplate assay as a rapid and inexpensive colorimetric method for determination of antibiotic susceptibility of Mycobacterium tuberculosis. J Clin Microbiol 2002; 40: 1873-1874.

24 Huang TS, Tu HZ, Lee SS, et al. Antimicrobial susceptibility testing of Mycobacterium tuberculosis to first-line drugs: comparisons of the MGIT 960 and BACTEC 460 systems. Ann Clin Lab Sci 2002; 32: 142-147.

25 Caviedes L, Lee TS, Gilman RH, et al. Rapid, efficient detection and drug susceptibility testing of Mycobacterium tuberculosis in sputum by microscopic observation of broth cultures. The Tuberculosis Working Group in Peru. J Clin Microbiol 2000; 38: 1203-1208.

26 Park WG, Bishai WR, Chaisson RE, et al. Performance of the microscopic observation drug susceptibility assay in drug susceptibility testing for Mycobacterium tuberculosis. J Clin Microbiol 2002; 40: 4750-4752.

27 Del Portillo P, Thomas MC, Martinez E, et al. Multiprimer PCR system for differential identification of mycobacteria in clinical samples. J Clin Microbiol 1996; 34: 324-328.

28 Canetti G, Fox W, Khomenko A, et al. Advances in techniques of testing mycobacterial drug sensitivity, and the use of sensitivity tests in tuberculosis control programmes. Bull World Health Organ 1969; 41: 21-43.

29 Canetti G, Froman S, Grosset J, et al. Mycobacteria: laboratory methods for testing drug sensitivity and resistance. Bull World Health Organ 1963; 29: 565-578.

30 Adjers-Koskela K, Katila ML. Susceptibility testing with the manual Mycobacteria Growth Indicator Tube (MGIT) and the MGIT 960 system provides rapid and reliable verification of multidrug-resistant tuberculosis. J Clin Microbiol 2003; 41: 1235-1239.

31 Fegou E, Jelastopulu E, Nicolaou S, et al. Comparison of the manual Mycobacteria Growth Indicator tube and the Etest with the method of proportion for susceptibility testing of Mycobacterium tuberculosis. Chemotherapy 2006; 52: 174-177.

32 Siddiqi SH, Hawkins JE, Laszlo A. Interlaboratory drug susceptibility testing of Mycobacterium tuberculosis by a radiometric procedure and two conventional methods. J Clin Microbiol 1985; 22: 919-923.

33 Roberts GD, Goodman NL, Heifets L, et al. Evaluation of the BACTEC radiometric method for recovery of mycobacteria and drug susceptibility testing of Mycobacterium tuberculosis from acidfast smear-positive specimens. J Clin Microbiol 1983; 18: 689-696. 
34 Bergmann JS, Woods GL. Reliability of mycobacteria growth indicator tube for testing susceptibility of Mycobacterium tuberculosis to ethambutol and streptomycin. J Clin Microbiol 1997; 35: 3325-3327.

35 Parsons LM, Somoskovi A, Urbanczik R, et al. Laboratory diagnostic aspects of drug resistant tuberculosis. Front Biosci 2004; 9: 2086-2105.

36 McDermott W, Tompsett R. Activation of pyrazinamide and nicotinamide in acidic environments in vitro. Am Rev Tuberc 1954; 70: 748-754
37 Butler WR, Kilburn JO. Susceptibility of Mycobacterium tuberculosis to pyrazinamide and its relationship to pyrazinamidase activity. Antimicrob Agents Chemother 1983; 24: 600-601.

38 Salfinger M, Heifets LB. Determination of pyrazinamide MICs for Mycobacterium tuberculosis at different $\mathrm{pHs}$ by the radiometric method. Antimicrob Agents Chemother 1988; 32: 1002-1004.

39 Kam KM, Sloutsky A, Yip CW, et al. Determination of critical concentrations of second-line anti-tuberculosis drugs with clinical and microbiological relevance. Int J Tuberc Lung Dis 2010; 14: 282-288. 